\title{
Change Management:
}

\section{warum und wozu?}

\author{
Anne-Geneviève Bütikofer
}

lic. iur., Generalsekretärin der FMH

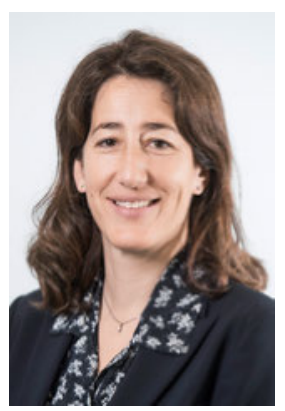

Jedes Unternehmen und jede Organisation - ob klein oder gross, öffentlich oder privat, familiär oder institutionell - durchläuft immer wieder Phasen des Wandels. Privat- und Gemeinschaftspraxen, die Institutionen und das Generalsekretariat der FMH - alle sind betroffen. In unserem beruflichen Alltag ist Change Management omnipräsent. Dies kann eine Umgestaltung des Arbeitsplatzes, die Einführung neuer Arbeitsinstrumente oder Formen der Zusammenarbeit (Digitalisierung) bedeuten, aber auch Massnahmen zur Steigerung des Wohlbefindens am Arbeitsplatz, zum Ausbau der Führungskompetenzen oder zur Anerkennung und Nutzung von Fachkompetenzen.

\section{Change Management ist ein notwendiger und kontinuierlicher Prozess.}

Die Effizienz und die Leistungsfähigkeit unserer Strukturen hängen von unserer Fähigkeit ab, Veränderungen ohne negative Folgen für unsere Mitarbeitenden und deren Arbeit umzusetzen. Ganz im Gegenteil sollten Veränderungen positive Auswirkungen zeigen. Erfolgreiches Change Management betrifft zuallererst den Menschen und ist vor allem auf diesen angewiesen, um von Erfolg gekrönt zu sein. Veränderungen verlangen eine enge Begleitung der betroffenen Personen, um ihnen die Anpassung zu erleichtern. Veränderungsprozesse ohne Erklärung, Kommunikation, Unterstützung und Schulung sind zum Scheitern verurteilt, da sich die Mitarbeitenden so nicht einbringen und mit den neuen Prozessen identifizieren können.

In einer Zeit, in welcher der Gesundheitsbereich unter erheblichem Druck steht, die wirtschaftlichen Bedingungen angespannt und die Kunden immer besser informiert sind (seien dies die Patienten gegenüber den Ärzten oder die Mitglieder gegenüber dem Generalsekretariat der FMH), muss das Change Management ein anpassungsfähiger und kontinuierlicher Prozess sein, damit sich die Mitarbeitenden bestmöglich entfalten und die an sie gerichteten Erwartungen erfüllen können.

Auch das Generalsekretariat der FMH stellt sich laufend der Notwendigkeit von Veränderungen und Anpassun-

gen. Wenn die Verantwortung für die Umsetzung des Change Managements bei der operativen Leitung liegt, dann muss sie sowohl von den Organen der FMH, dem Zentralvorstand und der Ärztekammer, als auch intern vom Kader unterstützt werden. Von Letzterem hängt der Erfolg zu einem wesentlichen Teil ab, da es die Mitarbeitenden am besten kennt und in der Lage ist, die mit Veränderungen verbundenen Risiken einschliesslich des sich dagegen bildenden «Veränderungsunwillens» zu erkennen und abzufedern. Aber auch die interne Kommunikation, die Personalabteilung und die Verantwortlichen für die Digitalisierung spielen eine zentrale Rolle bei der Umsetzung. Tatsächlich leisten digitale Hilfsmittel einen nicht zu unterschätzenden Beitrag zum Change Management, wenn sie im Rahmen einer genau definierten Strategie zum Einsatz kommen.

Wenn wir also Fortschritte erzielen wollen, müssen wir ein offeneres und flexibleres Arbeitsumfeld schaffen, das den Mitarbeitenden mehr Freiheit und Autonomie bietet. Um effizient zu sein, braucht es noch mehr Transparenz und eine noch bessere Zusammenarbeit mit unseren Mitarbeitenden. Dazu benötigen wir ein menschenorientiertes Management. Wir müssen sicherstellen, dass die neuen Technologien uns eine optimale Kommunikation und Raum für Kreativität schaffen. Gleichzeitig sollen die gemeinsamen Werte, das gegenseitige Vertrauen und das Wohlbefinden gestärkt werden. Dies bringt uns eine bessere Anpassungsfähigkeit an den Wechsel, mehr Freude an der Arbeit und schliess-

Um effizient zu sein, braucht es eine hohe Transparenz und eine gute Zusammenarbeit, aber auch gemeinsame Werte, gegenseitiges Vertrauen und Wohlbefinden.

lich eine deutlich bessere Effizienz. Dies ist also das Menu, welches das Generalsekretariat der FMH 2018 erwartet. Ich freue mich, diese Herausforderungen gemeinsam mit den Mitarbeitenden der FMH, deren Engagement auch 2017 wieder ausserordentlich war, angehen zu dürfen. Ich wünsche Ihnen schöne Feiertage und ein gutes neues Jahr mit vielen positiven Veränderungen. 